Sharif University of Technology
Scientia Iranica
SCIENTIA
I RAN I CA

Research Note

\title{
Optimum two-dimensional crack modeling in discrete least-squares meshless method by charged system search algorithm
}

\author{
H. Arzani ${ }^{a}$, A. Kaveh ${ }^{b, *}$ and M. Taheri Taromsari ${ }^{a}$ \\ a. Department of Civil Engineering, Shahid Rajaee Teacher Training University, Tehran, P.O. Box 16785-136, Iran. \\ b. Centre of Excellence for Fundamental Studies in Structural Engineering, Iran University of Science and Technology, Narmak, \\ Tehran, P.O. Box 16846-13114, Iran.
}

Received 14 March 2015; received in revised form 23 August 2015; accepted 21 December 2015

\author{
KEYWORDS \\ Discrete least-squares \\ meshless method; \\ Crack; \\ Error estimator; \\ Adaptive \\ rearrangement; \\ Charged system \\ search.
}

\begin{abstract}
In this paper, a node adaptive rearrangement is presented based on the estimated error in various domains for some problems in the fracture mechanics by Discrete Least-Squares Meshless method (DLSM). This method is one of the approximate methods recently introduced and used in the various fields. The method is based on minimization of the least-squares functional with respect to the nodal parameters, and it uses moving least-squares method for calculating the shape functions. Due to the natural process of problem solving, after calculating the shape functions, the residuals are calculated and their values are considered as an objective function for rearrangement of the nodes. There are three popular methods for constructing shape functions in discontinuous domains, and here, the transparency method is utilized. Similar to other numerical methods, there are different procedures for refinement and improvement of the results; however, adaptive rearrangement can be employed without increasing the computational cost. In this paper, the Charged System Search (CSS) algorithm is used as a tool for adaptive rearrangement or repositioning process. Efficiency and effectiveness of the proposed adaptive rearrangement technique is tested by some benchmark two-dimensional crack examples with available analytical solution around crack tips.
\end{abstract}

(C) 2017 Sharif University of Technology. All rights reserved.

\section{Introduction}

Finite Element Method (FEM) has been successfully used as a solution to a broad range of problems over the last decades. The method, however, encounters some difficulties when dealing with problems involving moving boundaries, crack propagation, or extremely large deformation due to their need for remeshing of the corresponding domains. These problems are still not fully resolved even with using refinement or

\footnotetext{
*. Corresponding author.

E-mail address: alikaveh@iust.ac.ir (A. Kaveh)
}

rearrangement procedures. Therefore, researchers have proposed a new method that uses some nodal points instead of meshing the problem domain [1-2]. Meshless methods are generally divided into two categories: the methods using weak form of differential equation and those utilizing strong form. One of the newest meshless methods that is based on strong form is discrete leastsquares meshless method [3]. Among many advantages of this method, no-need-for integration, symmetry of the coefficients matrix, and simplicity of application can be mentioned. This method and its variants have already been used to solve many problems in different fields [4-6]. It should be noted that for 
determining the nodes in the main node subdomain around discontinuous areas, it is necessary to consider some processes. There are three popular methods to do this [7-8], and each of them, considering some equations, introduces a way for including the nodes on other side of discontinuities. Due to the simplicity and effective transparency method, this procedure is used here.

There are many efficient and advanced mesh-free techniques which have been applied to complex discontinuous problems [9-12]. To improve the results of the numerical methods, including those of the DLSM, some refinement techniques are used. These methods are also divided into three categories: methods that increase the number of nodal points or make meshing finer (h-adaptivity); methods that increase degree of polynomial used for the shape functions (p-adaptivity); and those which use both prior methods simultaneously (hp-adaptivity). Adaptive methods can be found in the work of Afshar et al. [13] and Ebrahimnejad et al. [14].

In this paper, charged system search algorithm is used as a tool for node adaptive rearrangement in some problems related to fracture mechanics. This algorithm was developed by Kaveh and Talatahari [15] based on electrostatic and Newtonian mechanics laws. In CSS algorithm, some charged particles based on the objective function can attract or repeal each other. CSS has been used to optimize many problems in various fields such as optimum truss design [16], grillage system design [17], frame design [18], and problems in other fields of civil engineering [19-21], among others.

The aim of this paper is to present a simple and efficient way for node adaptive rearrangement in Discrete Least-Squares Meshless (DLSM) method for the problems that have high gradient answers in some domains. In Sections 2 and 4, the fundamental concepts of DLSM method and CSS algorithm are presented, respectively. In Section 3, transparency method is presented. In Section 5, error estimator and how to apply the CSS algorithm are discussed. In Section 6 , to demonstrate the effectiveness of the proposed techniques, some two-dimensional crack examples with analytical solution around crack tip are investigated, and also reduction of the residual in various domains is evaluated. Finally, some concluding remarks are presented in Section 7.

\section{Least-squares meshless}

\subsection{Moving least-squares shape functions}

Among the available meshless approximation schemes, the Moving Least-Squares (MLS) method [22] is generally considered to be one of the best methods to interpolate random data with a reasonable accuracy due to its completeness, robustness, and continuity [23,24]. With the MLS interpolation, unknown function, $\phi(x)$, is approximated by:

$$
\phi(x)=\sum_{i=1}^{m} P_{i}(x) . \alpha(x),
$$

where $P(x)$ is a polynomial basis in the space coordinates, and $m$ is the total number of the terms in the basis. For a $2 \mathrm{D}$ problem, we can specify $P(X)=$ [1 $x \quad y \quad x^{2} x y y^{2}$ ] for $m=6$. Here, $\alpha(x)$ is the vector of coefficients and can be obtained by minimizing a weighted discrete $L_{2}$ norm as follows:

$$
J=\sum_{i=1}^{n} W\left(x-x_{j}\right) \cdot\left[P^{T} \cdot \alpha(x)-\tilde{u}_{j}\right]^{2},
$$

where $n$ is the number of nodes in the domain and $\tilde{u}_{j}$ is the nodal value of the function to be approximated at point $x_{j}$. Weight function $W\left(x-x_{j}\right)$ is usually built in such a way that it has the following properties:

$W\left(x-x_{j}\right)>0$ within the support domain;

$W\left(x-x_{j}\right)=0$ outside the support domain;

$W\left(x-x_{j}\right) \quad$ monotonically decreases from the point of interest at $x$;

$W\left(x-x_{j}\right) \quad$ is sufficiently smooth, especially on the boundary of $\Omega_{j}$.

Here, the cubic spline weight function is employed for a better performance in meshless method as follows:

$$
\begin{aligned}
W\left(x-x_{j}\right) & =w(\bar{d}) \\
& = \begin{cases}\frac{2}{3}-4 \bar{d}^{2}+4 \bar{d}^{3} & \text { for } \bar{d} \leq \frac{1}{2} \\
\frac{4}{3}-4 \bar{d}+\bar{d}^{2}-\frac{4}{3} \bar{d}^{3} & \text { for } \frac{1}{2}<\bar{d} \leq 1 \\
0 & \text { fot } \bar{d}>1\end{cases}
\end{aligned}
$$

where $\bar{d}=\left(x-x_{j}\right) / d_{w}$, and $d_{w}$ is the size of influence domain of point $x_{j}$. Minimization of Eq. (2) with respect to coefficient $\alpha(x)$ leads to:

$$
\phi(x)=P^{T}(x) A^{-1}(x) B(x) \phi^{h},
$$

where:

$$
A(x)=\sum_{i=1}^{n} w\left(x-x_{j}\right) \cdot P\left(x_{j}\right) P^{T}\left(x_{j}\right),
$$

and:

$$
\begin{gathered}
B(x)=\left[w\left(x-x_{1}\right) P\left(x_{1}\right), w\left(x-x_{2}\right) P\left(x_{2}\right), \ldots,\right. \\
\left.w\left(x-x_{n}\right) P\left(x_{n}\right)\right] .
\end{gathered}
$$

Eq. (4) can be written in the following compact form:

$$
\phi(x)=\sum_{i=1}^{n} N_{i}^{T}(x) \phi_{i}(x)=N^{T}(x) \phi^{h},
$$

leading to the definition of MLS shape function defined as: 


$$
N^{T}(x)=P^{T}(x) A^{-1} B(x),
$$

where $N^{T}(x)$ contains the shape functions of nodes at point $x$ which are called Moving Least-Squares (MLS) shape functions.

\subsection{Discrete Least-Squares Meshless (DLSM) method}

Consider the following partial differential equation:

$$
\begin{cases}L(\phi)+f=0 & \text { in } \Omega \\ B(\phi)-\bar{t}=0 & \text { in } \Gamma_{t} \\ \phi-\bar{\phi}=0 \quad \text { in } & \Gamma_{u}\end{cases}
$$

where $L$ and $B$ are partial differential operators; $\bar{\phi}$ and $\bar{t}$ are vectors of prescribed displacements and tractions on the Dirichlet and Neumann boundaries, respectively; $\Omega$ is the considered domain; $\Gamma_{u}$ and $\Gamma_{t}$ are the displacement and traction boundaries, respectively; $f$ is the vector of external force or source term on the problem domain.

Suppose that the value of the estimating function $\phi$ at a point, such as $x_{k}$, is denoted as follows:

$$
\phi\left(x_{k}\right)=\sum_{i=1}^{m} N_{i}\left(x_{k}\right) \phi_{i}
$$

According to the discretization of the problem domain and its boundaries using Eq. (4), the residual of partial differential equation at point $x_{k}$ is defined as follows:

$$
R_{\Omega}\left(x_{k}\right)=L\left(\phi\left(x_{k}\right)\right)+f\left(x_{k}\right), \quad k=1, \ldots, M_{d} .
$$

The residual of Neumann boundary condition at point $x_{k}$ on the Neumann boundary can also be presented as:

$$
R_{t}\left(x_{k}\right)=B\left(\phi\left(x_{k}\right)\right)-\bar{t}\left(x_{k}\right), \quad k=1, \ldots, M_{t} .
$$

Finally, the residual of Dirichlet boundary condition at point $x_{k}$ on the Dirichlet boundary can be written as:

$$
R_{u}\left(x_{k}\right)=\phi-\bar{\phi}\left(x_{k}\right), \quad k=1, \ldots, M_{u},
$$

where $M_{d}$ is the number of internal points, $M_{t}$ is the number of points on the Neumann boundary, $M_{u}$ is the number of points on the Dirichlet boundary, and $M$ is the total number of points. A penalty approach can now be used to form the least-squares functional of the residuals defined as:

$$
J=\frac{1}{2}\left[\sum_{k=1}^{M_{d}} R_{\Omega}^{2}\left(x_{k}\right)+\alpha \cdot \sum_{k=1}^{M_{t}} R_{t}^{2}\left(x_{k}\right)+\beta \cdot \sum_{k=1}^{M_{u}} R_{u}^{2}\left(x_{k}\right)\right],
$$

where $\alpha$ and $\beta$ are the penalty coefficients for the Importance of Neumann and Dirichlet boundary conditions, respectively. Minimization of the functional with respect to nodal parameters $\left(\phi_{i}, i=\right.$ $1,2, \ldots, n)$ leads to the following system of equations:

$$
K \phi=F,
$$

where:

$$
\begin{aligned}
K_{i j}= & \sum_{k=1}^{M_{d}}[L(N)]_{k}^{T}[L(N)]_{k}+\alpha \sum_{k=1}^{M_{t}}[B(N)]_{k}^{T}[B(N)]_{k} \\
& +\beta \sum_{k=1}^{M_{u}} N_{k}^{T} N_{K}, \\
F_{t}= & \sum_{k=1}^{M_{d}}[L(N)]_{k}^{T}[L(N)]_{k} f_{k}+\alpha \sum_{k=1}^{M_{t}}[B(N)]_{k}^{T} \bar{t}_{k} \\
& +\beta \sum_{k=1}^{M_{u}}[N]_{k}^{t} \bar{\phi}_{k} .
\end{aligned}
$$

Stiffness matrix $K$ in Eq. (15) is square, symmetric, and positive definite. Therefore, the final system of equations can be solved directly via efficient solvers.

\section{Treatment of discontinuity in meshless methods}

Many engineering problems involve multi-connected domains and various kinds of discontinuities. For example, in elastostatics, for problems involving two materials, the coefficients in the partial differential equations are discontinuous across the interface between the materials. This results in solutions with discontinuous derivatives at the interface. When the approximation is a smooth function, such as the moving least-square approximation, the discontinuity in the derivative introduces spurious oscillations. Similarly, when a crack is modeled on a body, the dependent variable, i.e. the displacement, must be discontinuous across the crack. The introduction of discontinuity also requires special treatment in meshless methods [7]. Here, we introduce Transparency method for modeling discontinuities in DLSM.

\subsection{Transparency method}

In the transparency method [25], the shape function is smoothed around the tip of a discontinuity by endowing the surface, or line, of discontinuity with a varying degree of transparency. At the tip, the line of discontinuity is considered completely transparent, and the transparency diminishes as we move away 


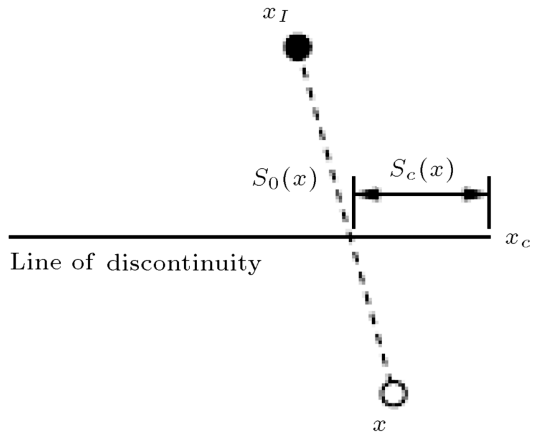

Figure 1. Schematic illustration of the transparency method for a node near a crack tip.

from the tip of the discontinuity. When a ray from evaluation point $x$ to node $x_{I}$ intersects with a line of discontinuity, parameter $s$ is modified (lengthened) by:

$$
S(x)=S_{0}(x)+S_{\max }\left(\frac{S_{C}(x)}{\bar{S}_{C}}\right)^{\lambda}, \quad \lambda \geq 2,
$$

where $S_{0}(x)$ is the distance between $x$ and $x_{I}, S_{\max }$ is the radius of the nodal support, and $S_{c}(x)$ is the distance from the crack tip to the intersection point (Figure 1). The parameter $\bar{S}_{C}$ sets the intersection distance at which the discontinuity line is completely opaque. Care should be exercised because, for nodes very close to a boundary, the angle enclosed by the crack and the ray from the node to the crack tip are very small, causing a sharp gradient in the weight functions. Therefore, all the nodes should have a minimum distance from the crack surface [8].

\section{Charged system search}

The charged system search has been developed by Kaveh and Talatahari [15] and applied successfully to many optimization problems. This method uses the electrostatics law of physics and Newtonian laws of mechanics. This method is a multi-agent optimization with agents being charged spheres. The Coulomb and Gauss laws provide the magnitude of the electric field at a point inside and outside a charged sphere, respectively, as follows:

$$
E_{i j}= \begin{cases}\frac{k_{e} q_{i}}{a^{3}} r_{i j} & \text { if } r_{i j}<a \\ \frac{k_{e} q_{i}}{r_{i j}^{2}} r_{i j} & \text { if } r_{i j} \geq a\end{cases}
$$

where $k_{e}$ is a constant known as the Coulomb constant; $r_{i j}$ is the separation of the centre of sphere and the selected point; $i q$ is the magnitude of the charge, and $a$ is the radius of the charged sphere. Using the superposition principle, the resulting electric force due to $N$ charged spheres is equal to [15]:

$$
\begin{gathered}
F_{j}=k_{e} q_{j} \sum_{i=1}^{N}\left(\frac{q_{i}}{a^{3}} r_{i j} i_{1}+\frac{q_{i}}{r_{i j}^{2}} i_{2}\right) \frac{r_{i}-r_{j}}{\left\|r_{i}-r_{j}\right\|}, \\
\left\{\begin{array}{l}
i_{1}=1, i_{2}=0 \Leftrightarrow r_{i j}<a \\
i_{1}=0, i_{2}=1 \Leftrightarrow r_{i j} \geq a
\end{array}\right.
\end{gathered}
$$

Also, according to Newtonian mechanics, we have [11]:

$$
\begin{aligned}
& \Delta r=r_{\text {new }}-r_{\text {old }}, \\
& V=\frac{r_{\text {new }}-r_{\text {old }}}{\Delta t}, \\
& a=\frac{V_{\text {new }}-V_{\text {old }}}{\Delta t},
\end{aligned}
$$

where $r_{\text {old }}$ and $r_{\text {new }}$ are the initial and final positions of a particle, respectively; $v$ is the velocity of the particle; and $a$ is the acceleration of the particle. Combining the above equations and using Newton's second law, the displacement of any object as a function of time is obtained as:

$$
r_{\text {new }}=\frac{1}{2} \frac{F}{m} \cdot \Delta t^{2}+V_{\text {old }} \cdot \Delta t+r_{\text {old }} .
$$

Inspired by the above electrostatics and Newtonian mechanics laws, the pseudo-code of the CSS algorithm is presented as follows [15]:

\section{Level 1: Initialization}

- Step 1. Initialization. Initialize the parameters of the CSS algorithm. Initialize an array of Charged Particles (CPs) with random positions. The initial velocities of the CPs are taken as zero. Each CP has a charge of magnitude $q$ defined considering the quality of its solution as:

$$
q_{i}=\frac{f i t(i)-\text { fitworst }}{\text { fitbest }- \text { fitworst }}, \quad i=1,2, \ldots, N
$$

where fitbest and fitworst are the best and the worst fitness of all the particles, and $f i t(i)$ represents the fitness of agent $i$. Separation distance $r_{i j}$ between two charged particles is defined as:

$$
r_{i j}=\frac{\left\|X_{i}-X_{j}\right\|}{\left\|\frac{X_{i}+X_{j}}{2}-X_{\text {best }}\right\|+\varepsilon}
$$

where $X_{i}$ and $X_{j}$ are the positions of $i$ th and $j$ th CPs, respectively; $X_{\text {best }}$ is the position of the best current $C$; and $\varepsilon$ is a small positive number used to avoid singularities;

- Step 2. CP ranking. Evaluate the values of the fitness function for the CPs, compare them with each other, and sort them in an increasing order;

- Step 3. CM creation. Store the number of the first CPs equal to Charged Memory Size (CMS) and their related values of the fitness functions in the Charged Memory (CM). 


\section{Level 2: Search}

- Step 1. Attracting force determination. Determine the probability of moving each $\mathrm{CP}$ toward the others considering the following probability function:

$$
P_{i j}= \begin{cases}1 & \frac{\text { fit }(i)-\text { fitbest }}{\text { fit }(i)-f i t(j)}>\text { rand } \bigvee \text { fit }(j)>f i t(i) \\ 0 & \text { else }\end{cases}
$$

and calculate the attracting force vector for each $\mathrm{CP}$ as follows:

$$
\begin{gathered}
F_{j}=q_{j} \sum_{i, i \neq j}^{N}\left(\frac{q_{i}}{a^{3}} r_{i j} \cdot i_{1}+\frac{q_{i}}{r_{i j}^{2}} \cdot i_{2}\right) p_{i j}\left(X_{j}-X_{i}\right) \\
\qquad\left\{\begin{array}{l}
j=1,2, \ldots, N \\
i_{1}=1, i_{2}=0 \Leftrightarrow r_{i j}<a \\
i_{1}=0, i_{2}=1 \Leftrightarrow r_{i j} \geq a
\end{array}\right.
\end{gathered}
$$

where $F_{j}$ is the resultant force affecting $j$ th $\mathrm{CP}$.

- Step 2. Solution construction. Move each CP to the new position and find its velocity using the following equations:

$$
\begin{aligned}
X_{j, \text { new }}= & \operatorname{rand}_{j 1} \cdot k a \cdot \frac{F_{j}}{m_{j}} \cdot \Delta t^{2}+\operatorname{rand}_{j 2} \cdot k_{v} \cdot V_{\text {old }} \cdot \Delta t \\
& +X_{j, \text { old }}, \\
V_{j, \text { new }}= & \frac{X_{j, \text { new }}-X_{j, \text { old }}}{\Delta t},
\end{aligned}
$$

where $\operatorname{rand}_{j 1}$ and $\operatorname{rand}_{j 2}$ are two random numbers uniformly distributed in range $(0,1) . \quad m_{j}$ is the mass of the CPs, which is equal to $q_{j}$ in this paper. The mass concept may be useful for developing a multi-objective CSS. $\Delta t$ is the time step, and it is set to $1 . k_{a}$ is the acceleration coefficient, and $k_{v}$ is the velocity coefficient to control the influence of the previous velocity.

- Step 3. CP position correction. If each CP exits from the allowable search space, correct its position using the HS-based handling approach as described for the HPSACO algorithm [24,26].

- Step 4. CP ranking. Evaluate and compare the values of the fitness function for the new CPs and sort them in an increasing order.

- Step 5. CM updating. If some new CP vectors are better than the worst ones in the $\mathrm{CM}$ in terms of their objective function values, then include the better vectors in the $\mathrm{CM}$ and exclude the worst ones from the CM.

\section{Level 3: Controlling the terminating criterion}

Repeat the search level steps until a terminating criterion is satisfied.

\section{Error indicator and adaptive rearrangement by CSS}

In the discrete least-squares meshless method for each nodal point, there are some nodes in its subdomain. The subdomains can be circular or rectangular (Figure 2). According to the value of shape function and using interpolation, the amount of residual for each node is calculated in both $x$ and $y$ directions. Then, by summing residual cube in $x$ and $y$ directions and getting square of it, the value of the total residual is calculated for each node and its subdomain nodes. Hence, for each node, a set of residuals will be attained for all subdomain nodes. The aim of this process is to reduce the residual related to main node, which this helps to reduce the amount of residual in various domains in the overall process.

General plane stress equations and the residuals are as follow:

$$
\left.\begin{array}{l}
\frac{\partial \sigma_{x}}{\partial x}+\frac{\partial \tau_{x y}}{\partial y}+F_{x}=0 \\
\Rightarrow \frac{\partial N}{\partial x} \sigma_{x}+\frac{\partial N}{\partial y} \tau_{x y}+F_{x}=e_{x} \\
\frac{\partial \tau_{x y}}{\partial y}+\frac{\partial \sigma_{y}}{\partial y}+F_{y}=0 \\
\Rightarrow \frac{\partial N}{\partial y} \sigma_{y}+\frac{\partial N}{\partial x} \tau_{x y}+F_{y}=e_{y}
\end{array}\right\} \rightarrow e=\sqrt{e_{x}^{2}+e_{y}^{2}}
$$

which they can be written in the matrix form as follows:

$$
\left[\begin{array}{ccc}
\frac{\partial N}{\partial x} & 0 & \frac{\partial N}{\partial y} \\
0 & \frac{\partial N}{\partial y} & \frac{\partial N}{\partial x}
\end{array}\right] \times\left[\begin{array}{c}
\sigma_{x} \\
\tau_{x y} \\
\sigma_{y}
\end{array}\right]+\left[\begin{array}{c}
F_{x} \\
F_{Y}
\end{array}\right]=\left[\begin{array}{c}
e_{x} \\
e_{Y}
\end{array}\right]
$$

To apply CSS algorithm, the main node and its subdomains node and the normalized value of residual are considered as CPs and their charges, respectively, as follows:

$$
q_{i}=\frac{e_{j}-e_{\min }}{e_{\max }-e_{\min }},
$$

where $e_{j}$ is the value of residual for $j$ th CP or $j$ th node, and $e_{\min }$ and $e_{\max }$ are the minimum and maximum values of residuals in the subdomain. Thus, the objective function for each node and its subdomain can be written as follows:

$$
\text { Minimize objective function }=\sum_{i=1}^{N} \operatorname{Residual}(i) \text {. }
$$

It should be noted that all of the programming processes are as identical to those mentioned in the CSS
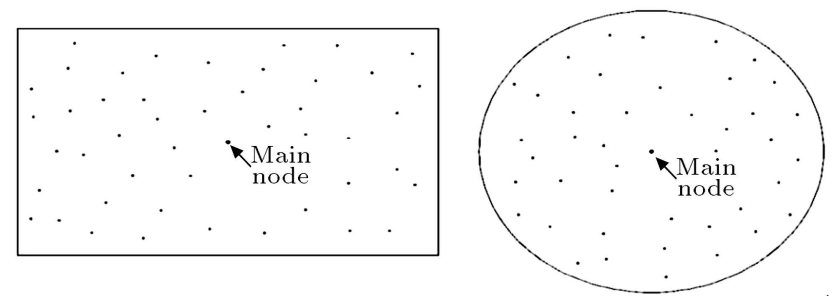

Figure 2. Nodes in the circular and rectangular subdomains. 
section; the only difference is considering mass factor for CPs which can be different due to the example type and value of the random parameters. In the examples discussed in this article, appropriate results are achieved with less than ten iterations. In addition, even by choosing a proper mass factor, this number can be reduced to 2 or 3 iterations.

\section{Numerical example}

In this section, three examples of different crack status are presented for which the analytical solutions are available around the crack tip [27-31]. These examples are in the plane stress state. A comparison between the results before and after the rearrangement and also investigations of the residual reduction in various domains show the effectiveness of the presented method. The first example is an edge crack, and the second and third ones are central and inclined crack, respectively, where uniaxial tension stress is applied to all of them.

\subsection{A plate with an edge crack}

This example is a plate with an edge crack under a uniaxial tension in the plane stress condition. The plate dimensions are $52 \times 20 \mathrm{~mm}$, and the crack length is $4 \mathrm{~mm}$ (Figure 3 ). The analytical solutions to this example and the next and [27-28] are obtained by the same Eqs. (35)-(38), but the only difference is stress factor that specifies which one is edge crack or central crack:

$$
\begin{aligned}
& \sigma_{x}=\frac{K_{I}}{\sqrt{2 \pi r}} \cos \frac{\theta}{2}\left[1-\sin \frac{\theta}{2} \sin \frac{3 \theta}{2}\right], \\
& \sigma_{y}=\frac{K_{I}}{\sqrt{2 \pi r}} \cos \frac{\theta}{2}\left[1+\sin \frac{\theta}{2} \sin \frac{3 \theta}{2}\right], \\
& \tau_{x y}=\frac{K_{I}}{\sqrt{2 \pi r}} \cos \frac{\theta}{2} \sin \frac{\theta}{2} \cos \frac{3 \theta}{2}, \\
& u=\frac{K_{I}}{G \sqrt{\frac{r}{2 \pi}}} \cos \frac{\theta}{2}\left[\frac{1-v}{1+v}+\sin ^{2} \frac{\theta}{2}\right], \\
& v=\frac{K_{I}}{G} \sqrt{\frac{r}{2 \pi}} \sin \frac{\theta}{2}\left[\frac{2}{1+v}-\cos ^{2} \frac{\theta}{2}\right],
\end{aligned}
$$

where $G$ is the shear module, $v$ representing the Poisson's ratio and $k_{I}$ is the stress factor, which can

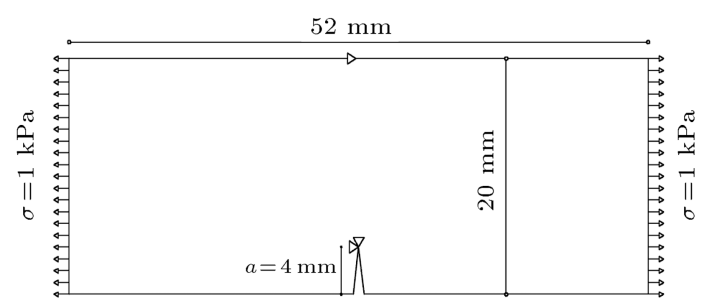

Figure 3. Edge crack examples boundary condition.

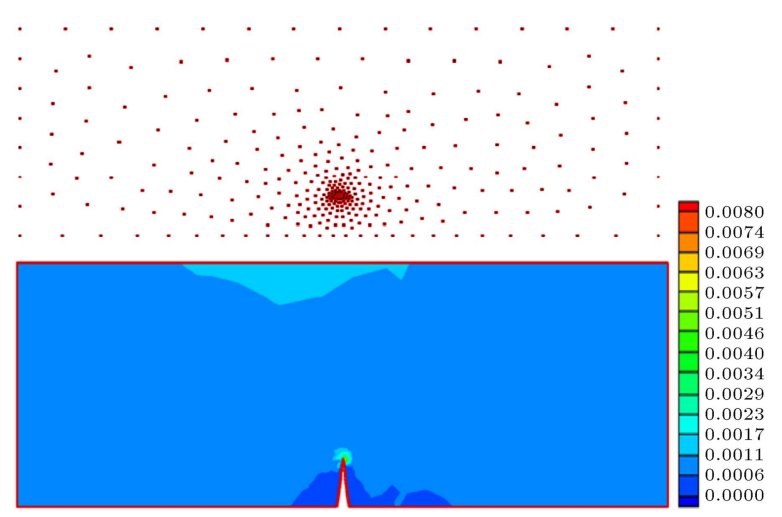

Figure 4. Initial nodes distribution and of contour.
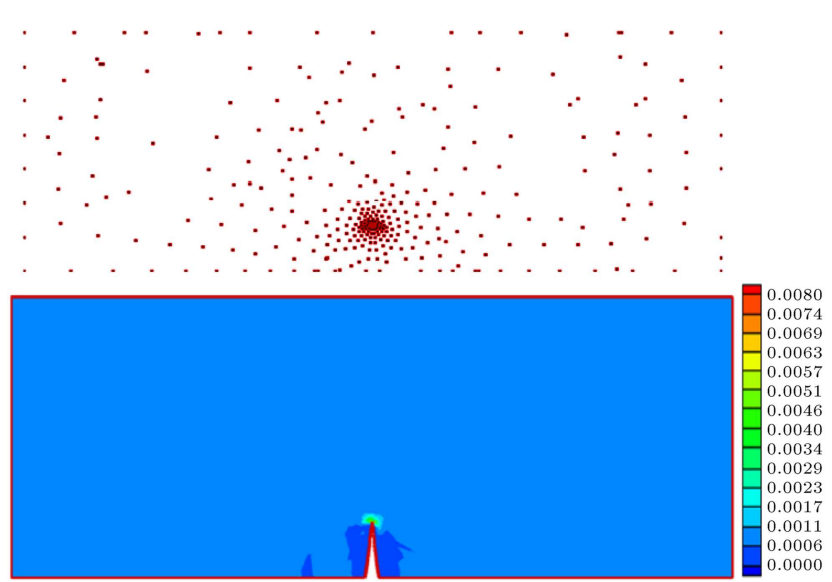

Figure 5. Nodes distribution and $\sigma_{x}$ contour after adaptive rearrangement by CSS.

be defined as:

$$
K_{I}=C \sigma \sqrt{\pi a},
$$

where $a$ is the crack length, and $C$ can define by as follows:

$$
\begin{aligned}
C= & 1.12-0.231\left(\frac{a}{2 b}\right)+10.55\left(\frac{a}{2 b}\right)^{2}-21.72\left(\frac{a}{2 b}\right)^{3} \\
& +30.39\left(\frac{a}{2 b}\right)^{4} .
\end{aligned}
$$

Here, $2 b$ is the Plane width. The process of simulation of this example begins with initial distribution of 314 nodes. The arrangements of nodes before and after the rearrangement are shown in Figures 4 and 5, respectively. After running CSS algorithm and comparing the obtained results for $\sigma_{x}$ along crack length, an improvement can be seen in the results. Average reduction in the residuals from ten successful runs in various problem domains is shown in Figures 6 and 7 .

\subsection{Plate with a central crack}

As a second example, a plate with dimensions of $32 \times 14 \mathrm{~mm}$ is loaded under uniform axial load, as shown 


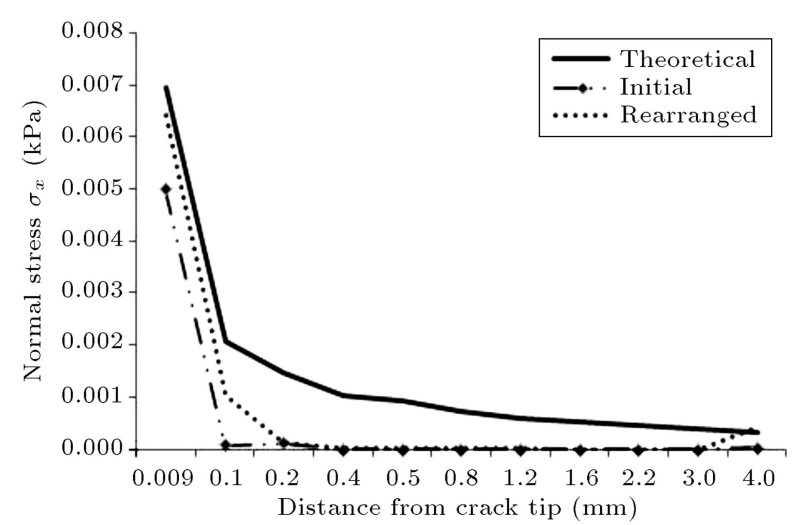

Figure 6. Normal stress $\sigma_{x}$ along crack.

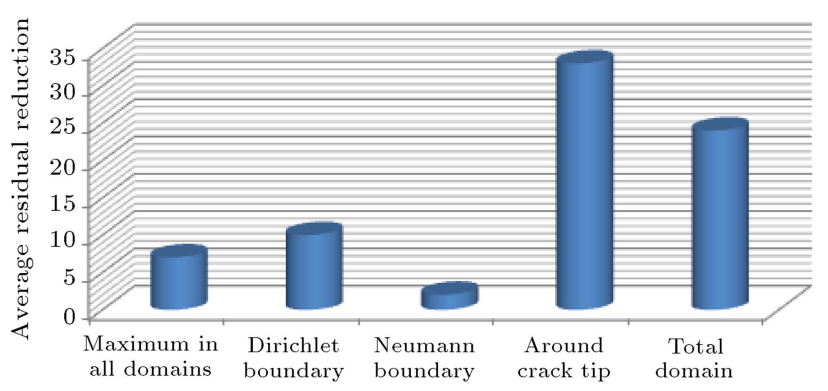

Figure 7. Average residual reduction from ten successful runs.

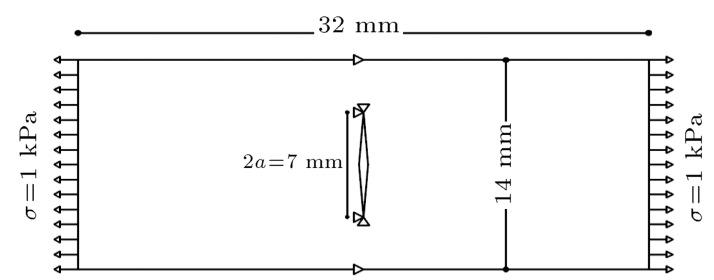

Figure 8. Central crack examples boundary conditions.

in Figure 8. Analytical solution to this can be defined by Eqs. (35)-(38) with a difference that the stress factor is obtained as [30]:

$$
K_{I}=\left(\frac{2 b}{\pi a} \tan \frac{\pi a}{2 b}\right)^{\frac{1}{2}}
$$

where $2 b$ and $2 a$ are the plate width and crack length, respectively.

This problem is solved with the initial configuration of 1418 nodal points (Figure 9). Then, by using CSS algorithm based on the value of residual in various problem domains, the nodes move toward the directions that the amount of error is more, and next, the problem is solved again by DLSM. This process is continued until the desired answer is achieved. Results show a good reduction in the residuals and also a good convergence with analytical solution around crack tip, as shown in Figures 10 and 11. The average residual reduction from ten successful runs is shown in Figure 12.

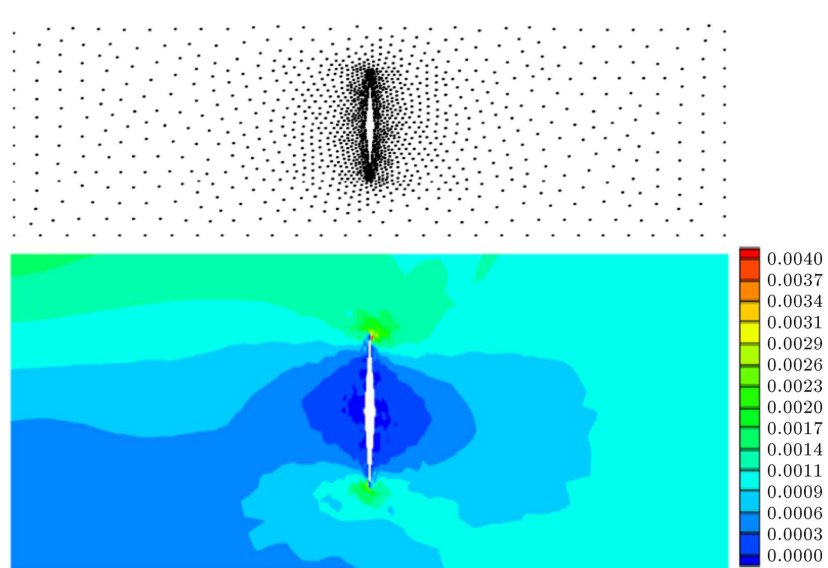

Figure 9. Initial nodes distribution and $\sigma_{x}$ contour.

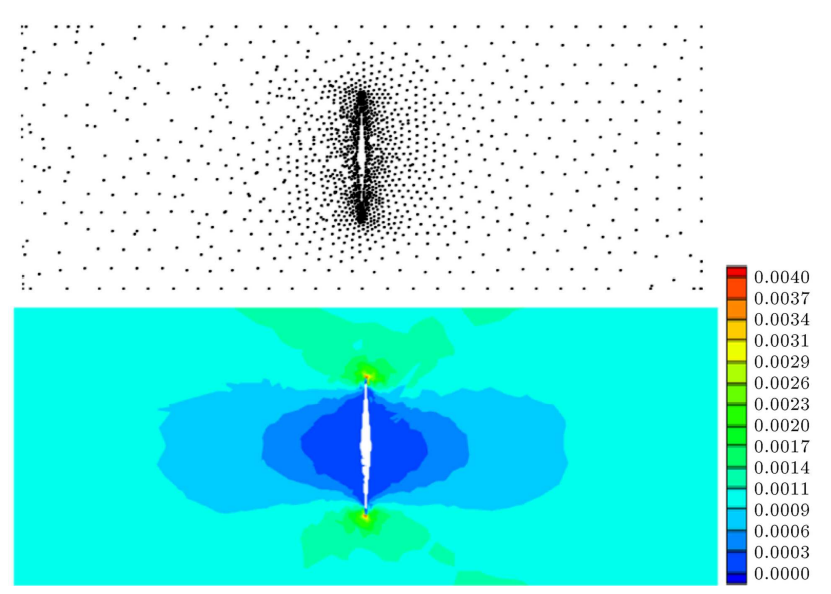

Figure 10. Nodes distribution and $\sigma_{x}$ contour after adaptive rearrangement by CSS.

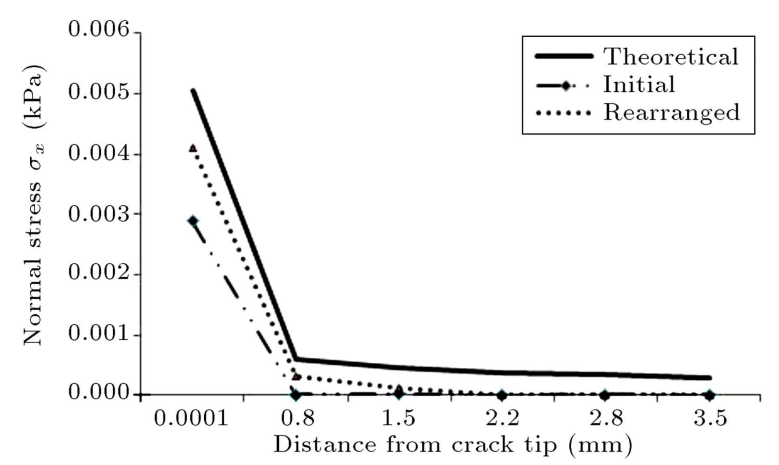

Figure 11. Normal stress $\sigma_{x}$ along crack.

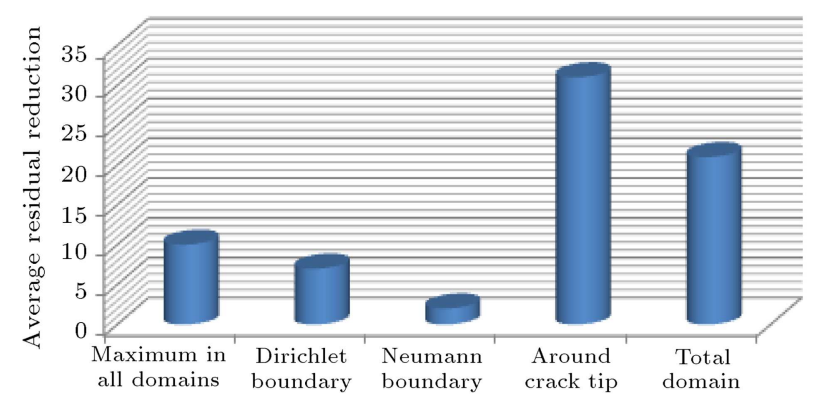

Figure 12. Average residual reduction from ten successful runs. 


\subsection{Plate with an inclined crack}

Previous examples are related to the first fracture mode means opening, but in the present example, combined fractured mode is investigated which involves opening and shear modes. Plate dimensions are similar to the second example and boundary conditions, as shown in Figure 13. Analytical solution for around crack tip is obtained as [30-31]:

$$
\begin{aligned}
\sigma_{x}= & \frac{K_{I}}{\sqrt{2 \pi r}} \cos \frac{\theta}{2}\left[1-\sin \frac{\theta}{2} \sin \frac{3 \theta}{2}\right] \\
& -\frac{K_{I I}}{\sqrt{2 \pi r}}\left[2+\cos \frac{\theta}{2} \cos \frac{3 \theta}{2}\right], \\
\sigma_{y}= & \frac{K_{I}}{\sqrt{2 \pi r}} \cos \frac{\theta}{2}\left[1+\sin \frac{\theta}{2} \sin \frac{3 \theta}{2}\right] \\
& +\frac{K_{I I}}{\sqrt{2 \pi r}} \sin \frac{\theta}{2} \cos \frac{\theta}{2} \cos \frac{3 \theta}{2} \\
\tau_{x y}= & \frac{K_{I}}{\sqrt{2 \pi r}} \cos \frac{\theta}{2} \sin \frac{\theta}{2} \cos \frac{3 \theta}{2}+\frac{K_{I I}}{\sqrt{2 \pi r}} \cos \frac{\theta}{2} \\
& {\left[1-\sin \frac{\theta}{2}-\sin \frac{3 \theta}{2}\right], } \\
u= & \frac{K_{I}}{G} \sqrt{\frac{r}{2 \pi}} \cos \frac{\theta}{2}\left[\frac{1}{2}(\kappa-1)+\sin ^{2} \frac{\theta}{2}\right] \\
& +\frac{K_{I I}}{G} \sqrt{\frac{r}{2 \pi}} \sin \frac{\theta}{2}\left[\frac{1}{2}(\kappa+1)+\cos ^{2} \frac{\theta}{2}\right] \\
v= & \frac{K_{I}}{G} \sqrt{\frac{r}{2 \pi}} \sin \frac{\theta}{2}\left[\frac{1}{2}(\kappa+1)-\cos ^{2} \frac{\theta}{2}\right] \\
& +\frac{K_{I I}}{G} \sqrt{\frac{r}{2 \pi}} \sin \frac{\theta}{2}\left[\frac{1}{2}(-\kappa+1)+\sin ^{2} \frac{\theta}{2}\right] .
\end{aligned}
$$

Here, $G$ is the shear modulus and $k=(3-v) /(1+v)$ with $v$ representing the Poisson's ratio. $\beta$ is the angle of crack line with $y$ axis; and $K_{I}$ and $K_{I I}$ are the stress factors for opening and shear modes based on crack angled, which can be defined as:

$$
\begin{aligned}
& K_{I}=\sigma \sqrt{\pi a} \sin ^{2} \beta, \\
& K_{I I}=\sigma \sqrt{\pi a} \sin ^{2} \beta \cos \theta .
\end{aligned}
$$

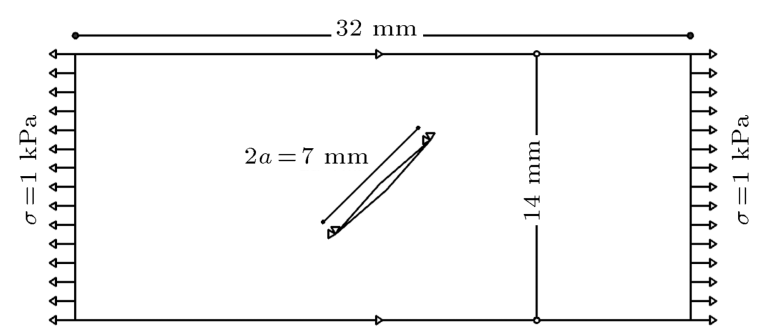

Figure 13. Inclined crack examples boundary conditions.

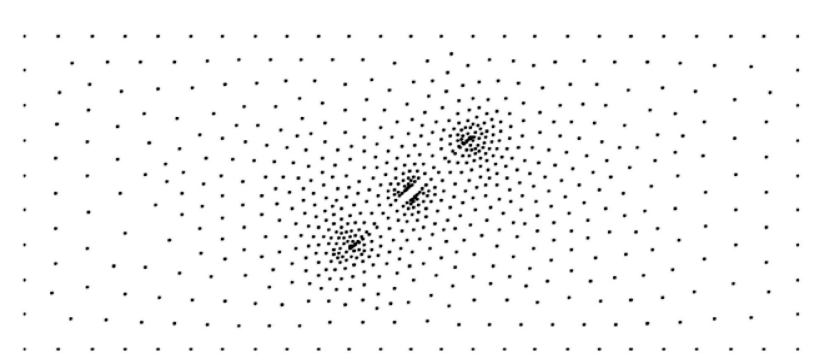

Figure 14. Initial nodes distribution.

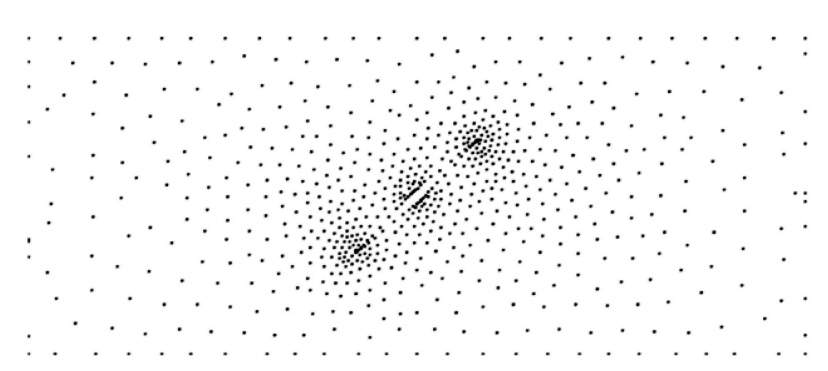

Figure 15. Nodes distribution after adaptive rearrangement by CSS.

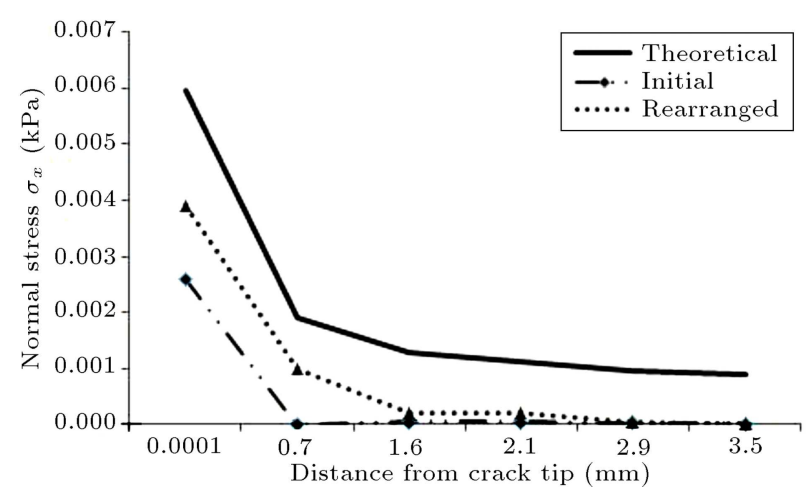

Figure 16. Normal stress $\sigma_{x}$ along crack.

The configuration begins with 752 nodal points (Figure 14), and then by using CSS algorithm, the nodes move adaptively (Figure 15). By comparing the $\sigma_{x}$ stress along crack before and after the rearrangement and also by investigating the residual reduction in problem domains, one can say adaptive rearrangement by these techniques has good influence on residual reduction and convergence with analytical solution (Figures 16 and 17).

\section{Conclusion}

In this paper, in order to obtain more accurate answers in discrete least-squares meshless method for solving problems in the field of fracture mechanics (twodimensional cracks), the CSS algorithm is used. Also, for determining the nodes with higher error, an error estimator is used. The amount of residual in each node and its subdomain nodes is considered as the charge of the CP, and then based on the CSS algorithm, the 


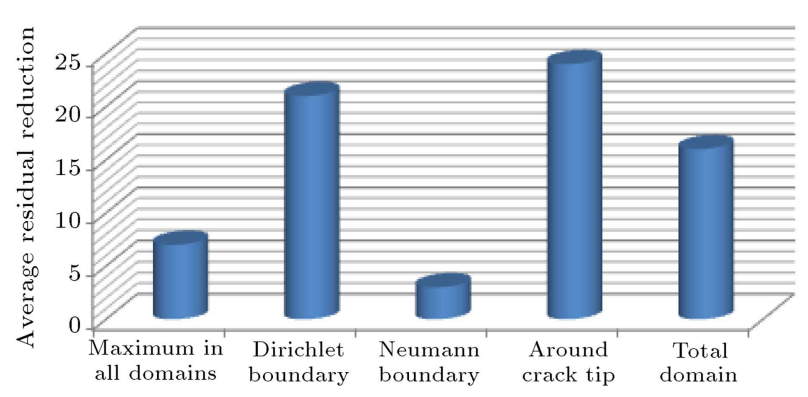

Figure 17. Average residuals reduction from ten successful runs.

nodes are moved in the direction where the amount of residual is higher. The results show the ability of the proposed method for solving problems in the field of fracture mechanics.

\section{References}

1. Pan, X.F., Sze, K.Y. and Zhang, X. "An assessment of the meshless weighted least-squares method", Acta Mechanica Solida Sinica, 17(3), pp. 270-282 (2004).

2. Liu, Y., Zhang, X. and Lu, M.W. "A meshless method based on least-squares approach for steadyand unsteady-state heat conduction problems", $\mathrm{Nu}$ merical Heat Transfer, Part B, 47(3), pp. 257-275 (2005).

3. Afshar, M.H. and Arzani, H. "Solving Poisson's equations by the discrete least squares meshless method", WIT Transaction on Modelling and Simulation, 42, pp. 23-32 (2004).

4. Firoozjaee, A. "Method for elasticity problems", International Journal of Civil Engineering, 7, pp. 9-18 (2009).

5. Afshar, M.H., Lashckarbolok, M. and Shobeyri, G. "Collocated discrete least squares meshless (CDLSM) method for the solution of transient and steady-state hyperbolic problems", International Journal for $\mathrm{Nu}$ merical Methods in Fluids, 60, pp. 1055-1078 (2009).

6. Chen, Y., Lee, J.D. and Eskandarian, A., Meshless Methods in Solid Mechanic, Springer Science \& Business Media, Inc (2006).

7. Liu, G.R., Mesh, R. and Afshar, M.H. "Discrete least squares meshless method with sampling points for the solution of elliptic partial differential equations", Engineering Analysis with Boundary Elements, 33(1), pp. 83-92 (2009).

8. Naisipour, M., Afshar, M.H., Hassani, B. and Firoozjaee, A.R., Collocation Discrete Least Square (CDLS) Free Methods Moving Beyond the Finite Element Method, CRC Press (2003).

9. Rabczuk, T., Zi, G., Bordas, S. and NguyenXuan, H. "A simple and robust three dimensional cracking-particle method without enrichment", Computer Methods in Applied Mechanics and Engineering, 199(37-40), pp. 2437-2455 (2010).
10. Rabczuk, T. and Belytschko, T. "Cracking particles: a simplified meshfree method for arbitrary evolving cracks", International Journal for Numerical Methods in Engineering, 61(13), pp. 2316-2343 (2004).

11. Rabczuk, T. and Zi, G. "A meshfree method based on the local partition of unity for cohesive cracks", Computational Mechanics, 39(6), pp. 743-760 (2007).

12. Rabczuk, T., Zi, G., Bordas, S. and Nguyen-Xuan, H. "A geometrically non-linear three dimensional cohesive crack method for reinforced concrete structures", Engineering Fracture Mechanics, 75(16), pp. 4740-4758 (2008).

13. Afshar, M.H., Amani, J. and Naisipour, M. "A node enrichment adaptive refinement in discrete least squares meshless method for solution of elasticity problems", Engineering Analysis with Boundary Elements, 36(3), pp. 385-393 (2012).

14. Ebrahimnejad, M., Fallah, N. and Khoei, A.R. "Adaptive refinement in the meshless finite volume method for elasticity problems", Computers and Mathematics with Applications, 69(12), pp. 1420-1443 (2015).

15. Kaveh, A. and Talatahari, S. "A novel heuristic optimization method: charged system search", Acta Mechanica, 213(3-4), pp. 267-286 (2010).

16. Kaveh, A. and Zolghadr, A. "Truss optimization with natural frequency constraints using a hybridized CSSBBBC algorithm with trap recognition capability", Computers and Structures, 102-103, pp. 14-27 (2012).

17. Kaveh, A. and Talatahari, S. "Charged system search for optimum grillage system design using the LRFDAISC code", Journal of Constructional Steel Res earch, 66, pp. 767-771 (2010).

18. Kaveh, A. and Talatahari, S. "Charged system search for optimal design of frame structures", Applied Soft Computing, 12, pp. 382-393 (2012).

19. Arzani, H., Kaveh, A. and Dehghan, M. "Adaptive node moving refinement in discrete least squares meshless method using charged system search", Scientia Iranica, 21(5), pp. 1529-1538 (2014).

20. ÖAzyön, S., Temurtas, H., Durmus, B. and Kuvat, G. "Charged system search algorithm for emission constrained economic power dispatch problem", Energy, 46, pp. 420-430 (2012).

21. Kaveh, A. and Behnam, A.F. "Cost optimization of a composite floor system, one-way waffle slab, and concrete slab formwork using charged system search algorithm", Scientia Iranica, 19(3), pp. 410-416 (2012).

22. Lancaster, P. and Salkauskas, K. "Surfaces generated by moving least squares method", Mathematics of Computation, 37, pp. 141-158 (1981).

23. Onate, E., Perazzo, F. and Miquel, J. "A finite point method for elasticity problems", Computers and Structures, 79, pp. 2151-2163 (2001).

24. Atluri, S.N. "The meshless local Petrov-Galerkin (MLPG) method for domain and boundary discretizations", Tech. Science Press (2004).

25. Organ, D., Fleming, M., Terry, T. and Belytschko, T. "Continuous meshless approximations for nonconvex 
bodies by diffraction and transparency", Computational Mechanics, 18, pp. 225-235 (1996).

26. Halliday, D., Resnick, R. and Walker, J. "Fundamentals of Physics", 8th Ed., John Wiley and Sons (2008).

27. Kaveh, A. and Talatahari, S. "Particle swarm optimizer, ant colony strategy and harmony search scheme hybridized for optimization of truss structures", Computers and Structures, 87(5-6), pp. 267-283 (2009).

28. Kaveh, A. and Talatahari, S. "A Particle swarm ant colony optimization algorithm for truss structures with discrete variables", Journal of Constructional Steel Research, 65(8-9), pp. 1558-1568 (2009).

29. Tada, H., Paris, P.C. and Irwin, G.R., The Stress Analysis of Crack Handbook, 3rd Ed., ASME Press (2000).

30. Sun, C.T. and Jin, Z.H., Fracture Mechanics, Academic Press (2012).

31. Miller, K.J. and McDowell, D.L. "Mixed-mode crack behavior", ASTM (1999).

\section{Biographies}

Hamed Arzani was born in 1969 in Amol, Iran. He obtained his BS, MS, and PhD degrees in Civil Engineering in University of Science and Technology in 1992, 1994, and 2006, respectively. He then joined the University of Shahid Rajaee Teacher Training University in Tehran, where he is presently an Assistant Professor of Structural Engineering. Arzani is the author of 3 papers published in international journals and 5 papers presented at international conferences.

Ali Kaveh was born in 1948 in Tabriz, Iran. After graduation from the Department of Civil Engineering in the University of Tabriz in 1969, he continued his studies on Structures at Imperial College of Science and Technology at London University and received his MS, DIC and PhD degrees in 1970 and 1974, respectively. He then joined the Iran University of Science and Technology in Tehran where he is presently a Professor of Structural Engineering. Professor Kaveh is the author of 485 papers published in international journals and 145 papers presented at international conferences. He has authored 23 books in Farsi and 7 books in English published by Wiley, the American Mechanical Society, Research Studies Press, and Springer.

Mojtaba Taheri Taramsari was born in 1988 in Rasht, Iran. He obtained his BS degree in Civil Engineering in Guilan University in 2011 and his MS degree in Structural Engineering at Shahid Rajaee Teacher Training University in 2014. 